\title{
Multiferroicity due to nonstoichiometry in the chain cuprate $\mathrm{LiVCuO}_{4}$
}

\author{
A.S. Moskvin ${ }^{1}$ and S.-L. Drechsler ${ }^{2}$ \\ 1 Ural State University, 620083 Ekaterinburg, Russia \\ ' ${ }^{2}$ Leibniz Institut für Festkörper- und Werkstoffforschung Dresden, D-01171, Dresden, Germany
}

PACS 74.72.-h - Cuprate superconductors (high- $T_{c}$ and insulating parent compounds)

PACS 75.10.Pq - Spin chain models

PACS 77.80.-e - Ferroelectricity and antiferroelectricity

\begin{abstract}
The recently observed multiferroic behavior in the $s=1 / 2$ chain cuprate $\mathrm{LiVCuO}_{4}$ $\left(\equiv \mathrm{LiCuVO}_{4}\right)$ with edge-shared $\mathrm{CuO}_{4}$ plaquettes and helical spin ordering does not agree with the existing theories such as a spin-current scenario. We argue that the effect can be consistently explained, if the exchange-induced electric polarization on the out-of-chain $\mathrm{Cu}^{2+}$ centers substituting for $\mathrm{Li}$-ions in $\mathrm{LiVCuO}_{4}$ is taken into account. These substituent centers are proved to be an effective probe of the spin incommensurability and the magnetic field effects.
\end{abstract}

The recent observations of multiferroic behaviour in edge-shared chain cuprates $\mathrm{LiVCuO}_{4}[1,2]$ and $\mathrm{LiCu}_{2} \mathrm{O}_{2}$ ' [3] challenge the multiferroic community. At first sight, these quantum $s=1 / 21 \mathrm{D}$ helicoidal magnets seem to be prototypical examples of 1D spiral-magnetic ferroelectrics revealing the "ferroelectricity caused by spin-currents" [4] with the textbook expression for the uniform polarization induced by a spin spiral with the wave vector $\mathbf{Q}$ :

$$
\mathbf{P} \propto\left[\mathbf{e}_{3} \times \mathbf{Q}\right],
$$

where $\mathbf{e}_{3}$ is a vector orthogonal to the spin spiral plane [5]. However, both $\mathrm{LiVCuO}_{4}$ and $\mathrm{LiCu}_{2} \mathrm{O}_{2}$ show up a behavior which is obviously counterintuitive within the framework of spiral-magnetic ferroelectricity $[2,3]$. Indeed, the spontaneous ferroelectric polarisation in $\mathrm{LiVCuO}_{4}$ is observed along the $a$-axis in agreement with the spin-current scenario. $P_{a}$ decreases with increasing external field along $a$-axis and vanishes for $h>2 \mathrm{~T}$, where the $b c$-plane helical structure is realized, however, without any signatures of the ferroelectricity along $c$-axis predicted by the existing theories. The saturated value of $P_{a}$ in $\mathrm{LiVCuO}_{4}$ in $h=0$ reveals a magnitude $\left(P_{a} \approx 43 \mu \mathrm{C} / \mathrm{m}^{2}\right)$ comparable with that of the multiferroic $\mathrm{Ni}_{3} \mathrm{~V}_{2} \mathrm{O}_{8}$ where the magnetic ordering drives the electric polarization $\approx 10^{2} \mu \mathrm{C} / \mathrm{m}^{2}$ (ref. [6]) that represents a typical magnitude of polarization induced by magnetic reordering in multiferroics. However, such an anomalously strong magnetoelectric effect seems to be an unexpexted feature for a system with $e_{g}$-holes and a nearly perfect highly symmetric chain struc-

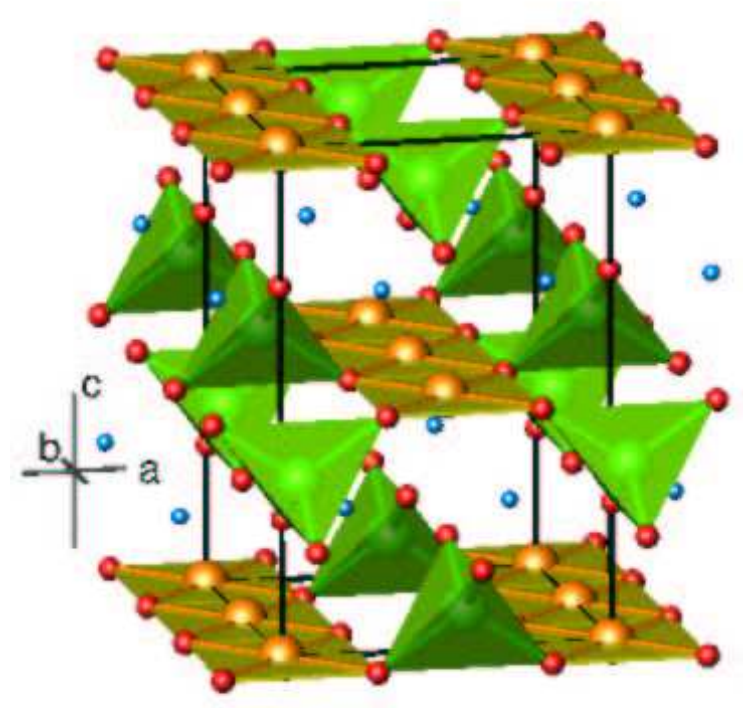

Fig. 1: (Color online). Crystal structure of $\mathrm{LiVCuO}_{4}$. (In the on-line colour version, the $\mathrm{O}^{2-}$ ions are highlighted in red, the $\mathrm{Li}^{+}$ions in blue, the $\mathrm{VO}_{4}$ tetrahedra are highlighted in green, the $\mathrm{CuO}_{2}$ chains in light olive).

ture (see Fig. 1) which both are unfavourable for a strong spin-electric coupling. Thus the magnetoelectric effect in the title cuprate $\mathrm{LiVCuO}_{4}$ raises fundamental questions about its microscopic origin.

Currently two essentially different spin structures of net 
electric polarization in crystals are considered: 1) with a bilinear nonrelativistic symmetric spin coupling [7-9]

$$
\mathbf{P}_{s}=\sum_{m n} \boldsymbol{\Pi}_{m n}^{s}\left(\mathbf{S}_{m} \cdot \mathbf{S}_{n}\right)
$$

or 2) a bilinear relativistic antisymmetric spin coupling $[4,5,10]$

$$
\mathbf{P}_{a}=\sum_{m n}\left[\boldsymbol{\Pi}_{m n}^{a} \times\left[\mathbf{S}_{m} \times \mathbf{S}_{n}\right]\right],
$$

respectively. The effective dipole moments $\Pi_{m n}^{s, a}$ depend on the $m, n$ orbital states and the $m n$ bonding geometry. First term stems somehow or other from a spin isotropic Heisenberg exchange interaction, see, e.g. ref. [11], and is not at work for $\mathrm{CuO}_{2}$ chains in $\mathrm{LiVCuO}_{4}$ because of inversion symmetry consideration. The second, or "spin-current" term is at present frequently considered to be one of the leading mechanisms of multiferroicity $[4,5,10,12-14]$. However, there are notable exceptions, in particular the manganites $\mathrm{RMn}_{2} \mathrm{O}_{5}, \mathrm{HoMnO}_{3}$, where a ferroelectric polarization can appear without any indication of magnetic chiral symmetry breaking $[7,9]$, and delafossite $\mathrm{CuFe}_{1-x} \mathrm{Al}_{x} \mathrm{O}_{2}$, where the helimagnetic ordering generates a spontaneous electric polarization $\|$ to the helical axis [15], in sharp contrast with the prediction of the spin current model. At closer examination the spin current mechanism comes to a dipole polarization of the threecenter $\mathrm{M}_{1}-\mathrm{O}-\mathrm{M}_{2}$ system due to an exchange-relativistic antisymmetric Dzyaloshinsky-Moriya (DM) type coupling with or without lattice degrees of freedom involved $[4,10]$. However, the original "spin-current" model by Katsura et al. [4] and its later versions [13,14] seem to be questionable as the authors proceed with an unrealistic scenario [16]. At variance with such a scenario one can apply a conventional procedure to derive an effective spin-operator $\hat{\mathbf{P}}_{a}=\left[\boldsymbol{\Pi}_{12}^{a} \times\left[\hat{\mathbf{S}}_{1} \times \hat{\mathbf{S}}_{2}\right]\right]$ for the electric dipole moment in the three-center $\mathrm{M}_{1}-\mathrm{O}-\mathrm{M}_{2}$ system like a technique suggested in refs. $[17,18]$ to derive expressions for the $\mathrm{Cu}$ and O spin-orbital contributions to the Dzyaloshinsky-Moriya coupling in cuprates. However, a straightforward application of this technique to the system of the $e_{g}$-holes in a perfect chain structure of edge-shared $\mathrm{CuO}_{4}$ plaquettes as in $\mathrm{LiVCuO}_{4}$ shows that the spin current does not produce an electric polarization [16]. An alternative mechanism of giant magnetoelectricity based on the antisymmetric DM type magnetoelastic coupling was proposed recently [10]. However, here we meet with a "weak" contributor. Indeed, the minimal value of $\gamma$ parameter $(\gamma=d \mathbf{D} / d \mathbf{R})$ needed to explain the experimental phase transition in multiferroic manganites is two orders of magnitude larger than reasonable microscopic estimates [10]. Thus we may state that the ideal edge-shared $\mathrm{CuO}_{4}$ plaquettes chain structure appears to be robust regarding the proper spin-induced electric polarization. It means that we should look for the origin of the puzzling multiferroicity in $\mathrm{LiVCuO}_{4}$ somewhere within the out-of-chain stuff.
In the Letter we argue that the ferroelectricity in this compound is actually unrelated to spin currents. It can be consistently explained if one takes into account the nonrelativistic effects of the spin-dependent electric polarisation [16] due to the out-of-chain $\mathrm{Cu}^{2+}$ centers substituting for Li-ions in $\mathrm{LiVCuO}_{4}$ present in real samples. For an illustration we start with a short description of a simple theory of the exchange-induced electric polarisation (2) which generalizes the model approach by Tanabe et al. [11]. For simplicity, let us consider an one-particle (electron/hole) center in a crystallographically centrosymmetric position of a magnetic crystal. Then all the particle states exhibit a definite spatial parity, even (g) or odd $(\mathrm{u})$, respectively. Having in mind the $3 \mathrm{~d}$ centers, we'll assume an even-parity ground state $|g\rangle$. For simplicity we restrict ourselves by only one excited odd-parity state $|u\rangle$. An isotropic exchange coupling with the surrounding spins can be written as follows:

$$
\hat{V}_{e x}=\sum_{n} \hat{I}\left(\mathbf{R}_{n}\right)\left(\mathbf{s} \cdot \mathbf{S}_{n}\right),
$$

where $\hat{I}\left(\mathbf{R}_{n}\right)$ is an orbital operator with a ma$\operatorname{trix}\left(\begin{array}{cc}I_{g g}\left(\mathbf{R}_{n}\right) & I_{g u}\left(\mathbf{R}_{n}\right) \\ I_{u g}\left(\mathbf{R}_{n}\right) & I_{u u}\left(\mathbf{R}_{n}\right)\end{array}\right)$. The parity-breaking offdiagonal part of exchange coupling can lift the center of symmetry and mix $|g\rangle$ and $|u\rangle$ states giving rise to a nonzero electric dipole polarization of the ground state

$$
\mathbf{P}=2 c_{g u}\langle g|e \mathbf{r}| u\rangle=\sum_{n} \mathbf{\Pi}_{n}\left(\mathbf{s} \cdot \mathbf{S}_{n}\right),
$$

where

$$
\boldsymbol{\Pi}_{n}=2 I_{g u}\left(\mathbf{R}_{n}\right) \frac{\langle g|e \mathbf{r}| u\rangle}{\Delta_{u g}} .
$$

and $\Delta_{u g}=\epsilon_{u}-\epsilon_{g}$.

It should be noted that at variance with the DM type, or the spin current scenario the direction of the exchangeinduced dipole moment for $i, j$ pair does not depend on the direction of the spins $\mathbf{S}_{i}$ and $\mathbf{S}_{j}$. In other words, the spin-correlation factor $\left\langle\mathbf{S}_{i} \cdot \mathbf{S}_{j}\right\rangle$ modulates a pre-existing dipole moment $\boldsymbol{\Pi}_{i j}$ which direction and value depend on the $\mathrm{Me}_{i}-\mathrm{O}-\mathrm{Me}_{j}$ bond geometry and the orbitals involved in the exchange coupling. The net exchange induced polarization of the magnetic crystal depends both on the crystal symmetry and the spin structure.

The orthorhombicaly distorted inverse spinel structure of $\mathrm{LiVCuO}_{4}$ [19] contains chains of edge-shared $\mathrm{LiO}_{6}$ and $\mathrm{CuO}_{6}$ octahedra running along $\mathbf{a}$ and $\mathbf{b}$ crystal axes, respectively [20]. The two neighboring $\mathrm{CuO}_{2}$ chains within the same $a b$ plane are connected by $\mathrm{VO}_{4}$ tetrahedra that alternate up and down along the chain direction, while two $\mathrm{CuO}_{2}$ chains within the same unit cell are connected by $\mathrm{LiO}_{6}$ octahedra (see Fig.1) First neutron-diffraction measurements [22] and a more detailed INS study [23] have revealed a long-range incommensurate helical magnetic ordering with a propagation vector $\mathbf{q}=(0,0.532,0)$ and moments confined to the $a b$-plane below $T_{N} \approx 2.1$ 
K., i.e. the spin spiral runs along the $b$-axis: $\mathbf{S}(y)=$ $S(\cos (\theta), \sin (\theta), 0)$ is the classical ground state of an isolated Heisenberg chain in $\mathrm{LiVCuO}_{4}$. Here $\theta=q y+\alpha, \alpha$ is a phase shift. The two $\mathrm{CuO}_{2}$ chains within the same unit cell are ordered antiferromagnetically: i.e. the phase shift $\Delta \alpha=\pi$. A combined NMR and AFMR study [24] also shows that in the low-field range $\mathrm{H}<\mathrm{H}_{c 1} \approx 2.5 \mathrm{~T}$, an $a b$-oriented planar spiral spin structure is realized in agreement with refs. 23,24. Based on NMR spectra simulations [24] and neutron scattering studies [2], the transition at $\mathrm{H}_{c 1}$ can be attributed to a spin-flop transition, where the spin plane of the magnetically ordered structure rotates to be perpendicular to the direction of the magnetic field applied in the easy plane.

Noteworthy, $\mathrm{LiVCuO}_{4}$ single crystals can reveal a sizeable non-stoichiometry with Li deficiency and appearance of $\mathrm{Cu}^{2+}$ or $\mathrm{Cu}^{3+}$ ions on native $\mathrm{Li}(4 d)$ positions [20] due to similar ionic radii of $\mathrm{Cu}$ and $\mathrm{Li}$. Indeed, a valence bond analysis [21] for such a $\mathrm{Cu}$ center with unrelaxed nearest neighbor $\mathrm{O}$ distances of $r_{1}=2.1 \AA$ and $r_{2}=2.18 \AA$ yields a valency $\nu_{C u}=\sum s_{C u-O} \approx 2$ :

$$
\nu_{C u}=4 \exp \left(\frac{r_{0}-r_{1}}{B}\right)+2 \exp \left(\frac{r_{0}-r_{2}}{B}\right)=1.798,
$$

where $r_{0}\left(\mathrm{Cu}^{2+}\right)=1.679 \AA$ and $B=0.37 \AA$. Adopting $r_{1}=2.05 \AA$ for the $\mathrm{Cu}-\mathrm{O}$ distances near the basal plane after a small relaxation of the adjacent unshared $\mathrm{O}$ ions above/below the $\mathrm{V}$ ions towards the $\mathrm{Cu}$ center one obtains $\nu_{C u}=1.98$. These numbers should be compared with $\nu_{C u}=2.16$ obtained for a regular $\mathrm{Cu}$ chain site.

Significant, up to $5 \%$ concentration of $\mathrm{Cu}$ ions on $\mathrm{Li}$ sites was assumed by Kegler et al. [25] to explain the appearance of an additional weak ${ }^{7} \mathrm{Li}$ NMR signal. The Li ions reside in two types of the centrosymmetrical positions A,B that differ by a bc-plane mirroring (see Fig. 1). Each $\mathrm{Li}$ ion has four nearest $\mathrm{Cu}^{2+}$ ions at the same distance. Below we argue that the $\mathrm{Cu}$ ions substituting for $\mathrm{Li}$ positions form strongly polarizable entities whose electric polarization due to a parity-breaking exchange interaction with the spin spirals in the chains is responsible for the multiferroicity in $\mathrm{LiVCuO}_{4}$.

Fig.2 shows both crystalline surrounding of the $\mathrm{Cu}^{2+}$ substituent center and oxygen-centered hole density distribution for active even-parity $b_{2 g}\left(\propto d_{x y}\right)$ and oddparity $e_{u}(\sigma)$ orbitals within the $\mathrm{D}_{4 h}$ point group. Let us consider the exchange-induced electric polarisation effects for these centers starting from a somewhat idealized "purely oxygen" model with active $\mathrm{O} 2 \mathrm{p} b_{2 g}$ and $e_{u}(\sigma)$ orbitals exchange-coupled with two $\mathrm{CuO}_{2}$ chains (see Fig.2). These orbitals are coupled by a large electric dipole matrix element:

$$
\left\langle b_{2 g}|e \mathbf{x}| e_{u y}(\sigma)\right\rangle=\left\langle b_{2 g}|e \mathbf{y}| e_{u x}(\sigma)\right\rangle=\frac{1}{\sqrt{2}} e R_{C u O},
$$

where $R_{C u O} \approx 2 \AA$ is the $\mathrm{Cu}-\mathrm{O}$ distance. Main exchange hole-hole coupling is determined by a "vertical" $\operatorname{pd} \pi$ transfer between O2px,y orbitals of central plaquette and $\mathrm{Cu}$
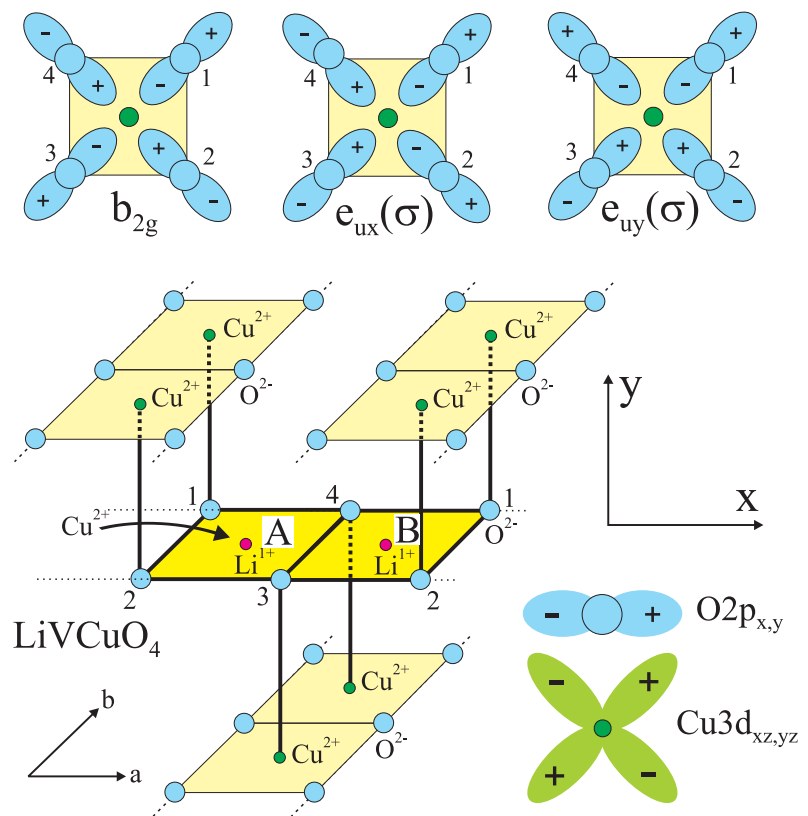

Fig. 2: (Color online). A slightly idealized view of the Li-O$\mathrm{Cu}$ bonds in the unit cell of $\mathrm{LiVCuO}_{4}$. An impurity center with a $\mathrm{Cu}$-ion substituted for $\mathrm{Li}$ is inbetween upper and lower $\mathrm{CuO}_{2}$ chains from the same unit cell. Top picture illustrates the hole density distributions for active even- and odd-parity O $2 p$ orbitals. Main hole $\mathrm{p}-\mathrm{d}$ transfer channel is shown.

$3 \mathrm{~d}_{x z, y z}\left(e_{g^{-}}\right)$orbitals of $\mathrm{Cu}$ ions in upper and lower chains. Diagonal $I_{g g}=I_{b_{2 g} b_{2 g}}$ and off-diagonal $I_{g u}=I_{b_{2 g} e_{u}}$ exchange integrals for e.g. $\mathrm{Cu}_{1}^{2+}-\mathrm{A}$ bond can be written as follows:

$$
\begin{gathered}
I_{g g}\left(C u_{n}\right)=t_{p d \pi}^{2}\left(\frac{1}{\Delta_{e_{g}}^{s}}-\frac{1}{\Delta_{e_{g}}^{t}}\right), \\
I_{g u x}(1)=I_{g u y}(1)= \\
\frac{1}{2} t_{p d \pi}^{2}\left(\frac{1}{\Delta_{e_{g}}^{s}}-\frac{1}{\Delta_{e_{g}}^{t}}+\frac{1}{\Delta_{e_{g}}^{s}-\Delta_{u g}}-\frac{1}{\Delta_{e_{g}}^{t}-\Delta_{u g}}\right),
\end{gathered}
$$

where $\Delta_{e_{g}}^{s}=\epsilon_{e_{g}}+A+B+2 C, \Delta_{e_{g}}^{t}=\epsilon_{e_{g}}+A-5 B$ are the energies of the two-hole copper singlet and triplet terms with $b_{2 g} e_{g}$ configurations, respectively, $\mathrm{A}, \mathrm{B}$, and $\mathrm{C}$ are Racah parameters, $\Delta_{u g}=\epsilon_{e_{u}}-\epsilon_{b_{2 g}}$. The exchange channel we consider results in a significant ferromagnetic $g g$ coupling between $\mathrm{Cu}^{2+}$-substituent and adjacent $\mathrm{CuO}_{4-}^{-}$ centers from lower and upper chains. However, the magnitude of the off-diagonal $g u$-exchange integrals can sufficiently exceed that of a conventional diagonal exchange integral mainly due to a smaller value of the energy separation $\left(\Delta_{e_{g}}^{s, t}-\Delta_{u g}\right)$ as compared with $\Delta_{e g}^{s, t}$. Simple symmetry considerations point to the following relations between exchange-dipole parameters $\boldsymbol{\Pi}_{n}$

$$
\begin{gathered}
\Pi_{1 x}=\Pi_{2 x}=-\Pi_{3 x}=-\Pi_{4 x}= \pm \Pi ; \\
\Pi_{1 y}=-\Pi_{2 y}=-\Pi_{3 y}=\Pi_{4 y}=\Pi,
\end{gathered}
$$



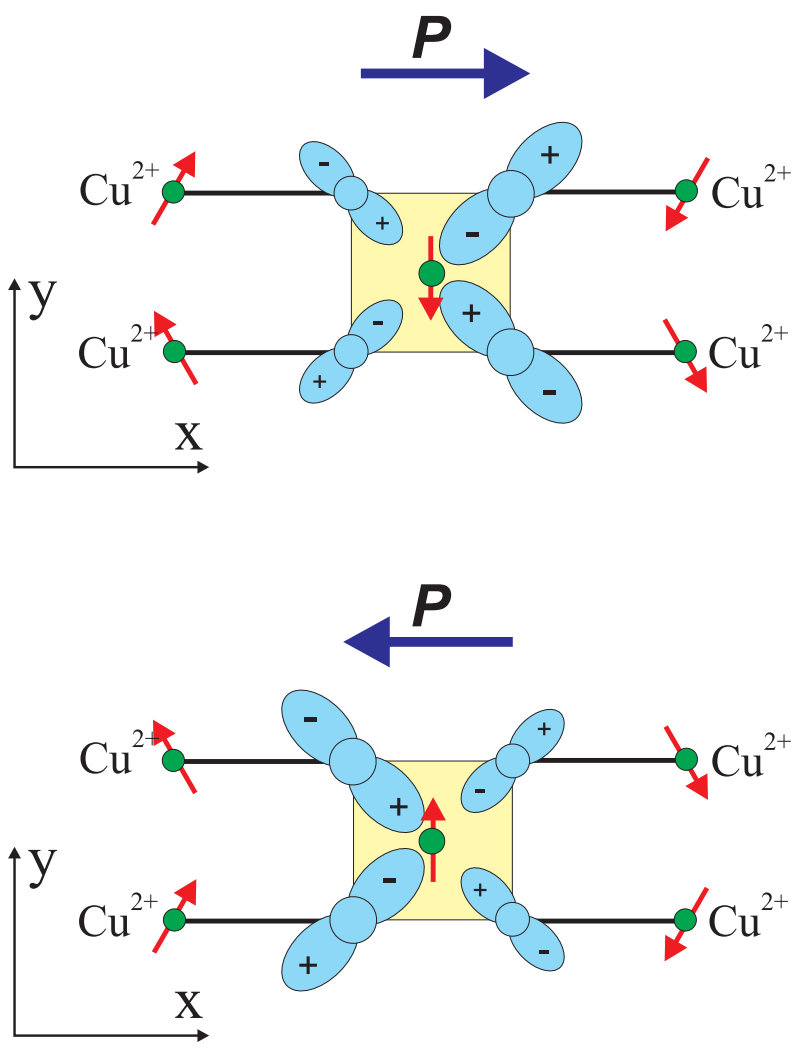

Fig. 3: (Color online). Illustration of the exchange-induced polarisation effect for a $\mathrm{Cu} 3 d \mathrm{O} 2 p b_{2 g}$ hole at a Li-position of $\mathrm{LiVCuO}_{4}$. The chain spiral $\mathrm{Cu}^{2+}$ spins are arranged to give nonzero $\mathrm{G}_{x}$ and $\mathrm{A}_{y}$ modes.

where upper and lower signs correspond to positions A and $\mathrm{B}$, respectively. Illustration of the exchange-induced polarization effect for $\mathrm{O} 2 \mathrm{p} b_{2 g}$ hole in Li-position of $\mathrm{LiVCuO}_{4}$ is presented in Fig. 3. It should be emphasized that the induced electric dipole moment is of purely electronic nature and lies in the $a b$ plane. To describe different configurations of the spin neighbourhood for a $\mathrm{Cu}^{2+}$ substituent (see Fig.22), we introduce four basic vectors similarly to conventional ferro- and antiferromagnetic vectors as follows:

$\mathbf{F}(y)=\left[\mathbf{S}_{1}+\mathbf{S}_{2}+\mathbf{S}_{3}+\mathbf{S}_{4}\right] ; \mathbf{G}(y)=\left[\mathbf{S}_{1}-\mathbf{S}_{2}+\mathbf{S}_{3}-\mathbf{S}_{4}\right] ;$

$\mathbf{A}(y)=\left[\mathbf{S}_{1}+\mathbf{S}_{2}-\mathbf{S}_{3}-\mathbf{S}_{4}\right] ; \mathbf{C}(y)=\left[\mathbf{S}_{1}-\mathbf{S}_{2}-\mathbf{S}_{3}+\mathbf{S}_{4}\right]$

( $y$ points to the substituent coordinate along a spin chain) with a kinematic constraint: $(\mathbf{F} \cdot \mathbf{A})=(\mathbf{C} \cdot \mathbf{G})=0$ valid for two identical spirals in the unit cell irrespective of phase shift. In terms of new vectors we arrive at simple expressions for local electric polarization in $\mathrm{A}$ and $\mathrm{B}$ positions:

$$
P_{a}(y)= \pm d_{a}(\mathbf{s} \cdot \mathbf{A}) ; P_{b}(y)=d_{a}(\mathbf{s} \cdot \mathbf{C}) .
$$

For two ferromagnetically ordered spirals (phase shift $\Delta \alpha=0) \mathbf{A}=\mathbf{G}=0$, while for two antiferromagnetically ordered spirals as in $\mathrm{LiVCuO}_{4}$ (phase shift $\Delta \alpha=\pi$ ) $\mathbf{F}=\mathbf{C}=0$. It means that the $b$-component of the electric polarisation $P_{b}(y)$ for a mutual antiferromagnetic (antiphase) spin ordering of the "upper" and the "lower" $\mathrm{CuO}_{2}$ chains $\left(\mathbf{S}_{1}=-\mathbf{S}_{4}, \mathbf{S}_{2}=-\mathbf{S}_{3}\right)$ vanishes: $P_{b}(y)=0$ irrespective of the spin-spiral plane orientation, and the substituent electric polarisation may be oriented only along crystal $a$-axis. Moreover, the nonzero net crystal polarisation $\left\langle P_{a}(y)\right\rangle$ one obtains only, if $\mathbf{s}_{A} \neq \mathbf{s}_{B}$, or for magnetically nonequivalent $\mathrm{A}$ and $\mathrm{B}$ substituent positions. Spin polarisation of the $\mathrm{Cu}^{2+}$ substituent spin can be easily found within the framework of a weak coupling approximation, if one takes the most general form of the impurity-spiral ground state $(g g)$ exchange interaction

$$
V_{s S}=\sum_{i=1-4} \hat{\mathbf{s}} \overleftrightarrow{\mathbf{I}}(\mathbf{i}) \hat{\mathbf{S}}_{i}=\left(\hat{\mathbf{s}} \cdot \hat{\mathbf{H}}_{0}\right)
$$

where $\hat{\mathbf{H}}_{0}$ is an effective magnetic field, acting on the $\mathrm{Cu}^{2+}$ substituent, $I_{\alpha \alpha}(i)=I_{\alpha \alpha}, I_{x z}(i)= \pm I_{x z} ; I_{x y}(1)=$ $-I_{x y}(2)=I_{x y}(3)=-I_{x y}(4)= \pm I_{x y} ; I_{z y}(1)=-I_{z y}(2)=$ $I_{z y}(3)=-I_{z y}(4)=I_{z y}$ form a symmetric matrix of the exchange integrals, the upper and lower signs correspond to positions A and B, respectively. Thus for effective field we obtain

$$
\mathbf{H}_{0}(y)=\stackrel{\leftrightarrow}{\mathbf{I}}_{\mathbf{F}} \mathbf{F}+\stackrel{\leftrightarrow}{\mathbf{I}}_{\mathbf{G}} \mathbf{G}
$$

with

$$
\stackrel{\leftrightarrow}{\mathbf{I}}_{\mathbf{F}}=\left(\begin{array}{ccc}
I_{x x} & 0 & \pm I_{x z} \\
0 & I_{y y} & 0 \\
\pm I_{x z} & 0 & I_{z z}
\end{array}\right) ; \stackrel{\mathbf{I}}{\mathbf{G}}_{\mathbf{G}}=\left(\begin{array}{ccc}
0 & \pm I_{x y} & 0 \\
\pm I_{x y} & 0 & I_{z y} \\
0 & I_{z y} & 0
\end{array}\right)
$$

Effective molecular field on the $\mathrm{Cu}^{2+}$ substituent spin in $\mathrm{LiVCuO}_{4}$ is determined solely by anisotropic terms due to a complete cancellation of isotropic contributions $(\mathbf{F}=0)$. Thus, the exchange-induced electric polarisation $P_{a}(y)$ for O2p $b_{2 g}$ hole in Li-position can be written as follows:

$$
\begin{gathered}
P_{a}(y)= \pm d_{a}(\mathbf{s} \cdot \mathbf{A})= \pm \frac{d_{a} S}{H(y)}\left(\mathbf{h}+\mathbf{H}_{0}\right) \cdot \mathbf{A}= \pm \frac{d_{a} S}{H(y)}(\mathbf{h} \cdot \mathbf{A}+ \\
\left. \pm I_{x y}\left(G_{x} A_{y}+G_{y} A_{x}\right)+I_{z y}\left(G_{z} A_{y}+G_{y} A_{z}\right)\right)
\end{gathered}
$$

where $d_{a}=\sqrt{2} e R_{C u O} \Delta_{u g}^{-1} I_{b_{1 g} e_{u x}(\sigma)}\left(C u_{1}\right), \mathbf{h}$ is an external magnetic field. It is worth noting that the effective magnetic field in these expressions reflects the symmetry of conventional $(g g)$ exchange coupling, while the basic spin vectors $\mathbf{A}$ and $\mathbf{C}$ do that of the parity-breaking $(u g)$ exchange coupling.

We start with an $a b$-planar spiral as the zero-field ground state of the $\mathrm{CuO}_{2}$ chains in $\mathrm{LiVCuO}_{4}$ and assume $\mathrm{T}=0$. Then the nonzero basic spin vectors and the resultant magnetic field acting on the spin of the $\mathrm{Cu}^{2+}$ substituent at $y$-position can be written as follows:

$$
\begin{gathered}
G_{x}=-4 S \sin \left(\frac{q b}{2}\right) \sin (q y) ; G_{y}=4 S \sin \left(\frac{q b}{2}\right) \cos (q y) ; \\
A_{x}=4 S \cos \left(\frac{q b}{2}\right) \cos (q y) ; A_{y}=4 S \cos \left(\frac{q b}{2}\right) \sin (q y) . \\
H_{x}=h_{x}+H_{0 x}=h_{x} \pm 4 S I_{x y} \sin \left(\frac{q b}{2}\right) \cos (q y) ;
\end{gathered}
$$




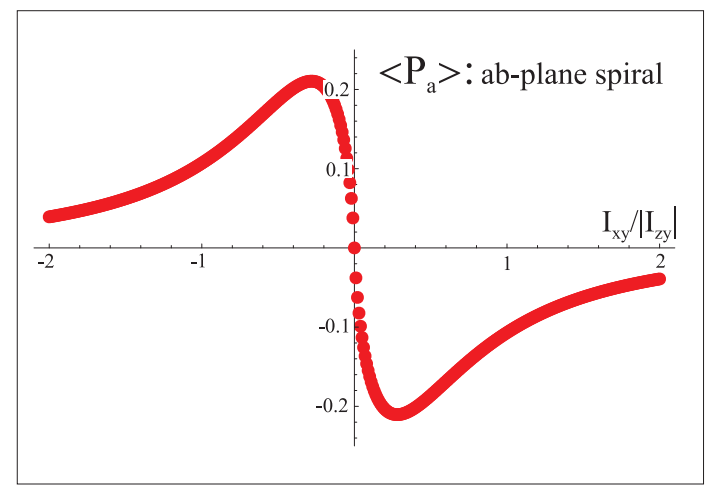

Fig. 4: (Color online). The dependence of the mean value of the $a$-axis electric polarisation of the $\mathrm{Cu}^{2+}$ substituent $\left\langle P_{a}(y)\right\rangle$ (in units of $d_{a} /$ ion) on the ratio of exchange parameters $I_{x y} / I_{z y}$.

$$
\begin{gathered}
H_{y}=h_{y}+H_{0 y}=h_{y} \mp 4 S I_{x y} \sin \left(\frac{q b}{2}\right) \sin (q y) ; \\
H_{z}=h_{z}+H_{0 z}=h_{z}+4 S I_{z y} \sin \left(\frac{q b}{2}\right) \cos (q y),
\end{gathered}
$$

We see that both all the components and absolute value of effective magnetic field oscillate along the chain direction in a complex way thus providing at variance with the DM type mechanism the oscillatory behavior of electric polarisation accompanying the spin spiral ordering. Electric polarisation $P_{a}(y)$ oscillates along the chain direction $b$ as follows:

$$
\begin{aligned}
P_{a}(y)= \pm & \frac{2 d_{a} S \cos \left(\frac{q b}{2}\right)}{H(y)}\left[h_{x} \cos (q y)+h_{y} \sin (q y)\right. \\
& \left. \pm 2 I_{x y} \sin \left(\frac{q b}{2}\right) \cos (2 q y)\right] .
\end{aligned}
$$

Interestingly, the change of the spiral modulation wave length, inversely proportional to the pitch angle $(q b)$, should affect the magnitude of the electric polarisation. Formally, the spontaneous $(h=0)$ zero-temperature polarisation $P_{a}(y)$ does depend on the $I_{x y} /\left|I_{z y}\right|$ ratio rather than on the numerical values of exchange anisotropy parameters. It changes sign with that of $I_{x y}$. At the same

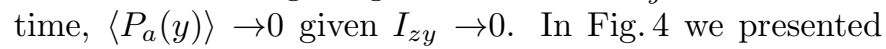
the dependence of the mean value of the $a$-axis electric polarisation of the $\mathrm{Cu}^{2+}$ substituent $\left\langle P_{a}(y)\right\rangle$ (in units of $d_{a} /$ ion) given $q b / 2=0.233 \pi$ that corresponds to a pitch angle $\left.\approx 84^{\circ}[22,23]\right)$ on the $I_{x y} / I_{z y}$ ratio that points to a significance of both integrals in the magnetoelectric effect.

We see that for a reasonable ratio between the exchange integrals $I_{x y}$ and $I_{z y}$ the averaging procedure can significantly reduce the local polarisation from several times to more than one order of magnitude. Maximal magnitudes of polarisation one obtains for $\left|I_{x y} / I_{z y}\right| \approx 0.4$. Fig. [5 shows the field dependence of the mean value of the $a$ axis electric polarisation of the $\mathrm{Cu}^{2+}$ substituent $\left\langle P_{a}(y)\right\rangle$ in frames of a rigid $a b$-plane spiral approximation given $I_{x y} / I_{z y}=0.5$. In other words, we assume the external magnetic field does not distort the spin spiral, however,

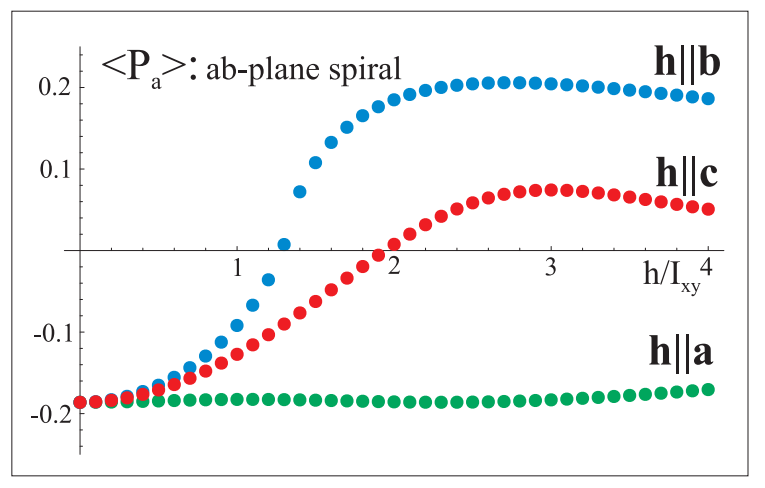

Fig. 5: (Color online). The field dependence of the mean value of the $a$-axis electric polarisation of the $\mathrm{Cu}^{2+}$ substituent $\left\langle P_{a}(y)\right\rangle$ (in units of $d_{a} /$ ion) for $a b$-plane spiral: $I_{x y} / I_{z y}=0.5$.

polarises the substituent spin. It is worth noting that the averaging procedure should be performed carefully, as we deal with a very slow convergence of the chain summing.

For the $b c$-plane spin spiral ordering the electric polarisation $P_{a}(y)$ oscillates both for $\mathrm{A}$ and $\mathrm{B}$ substituent positions with a magnitude which can be easily obtained from (16), if one makes the interchange: $h_{x} \rightarrow h_{z}, \pm I_{x y} \leftrightarrow I_{z y}$. In other words, it means that in a zero magnetic field $\left.P_{a}\left(I_{x y} / I_{z y}\right)\right|_{a b}=\left.P_{a}\left(I_{z y} / I_{x y}\right)\right|_{b c}$. However, at variance with the $a b$-plane spiral, here we deal with an exact compensation of A- and B-contributions to the net electric polarisation $\left\langle P_{a}(y)\right\rangle:\left\langle P_{a}(y)\right\rangle_{A}=\left\langle P_{a}(y)\right\rangle_{B}=0$.

For the $a c$-plane spiral the molecular field $\mathbf{H}_{0}$ on the copper substituent is directed along the $\mathbf{b}$-axis, hence, in accordance with (13) and in absence of external magnetic field $P_{a}(y)$ vanishes. The external magnetic field applied in $a c$-plane induces the oscillatory electric polarisation, however, the resultant polarisation $\left\langle P_{a}(y)\right\rangle$ vanishes irrespective of the field direction.

Thus the spin-spiral ordering in the $\mathrm{CuO}_{2}$ chains of $\mathrm{LiVCuO}_{4}$ does induce due to the parity-breaking exchange a spontaneous electric polarisation on the $\mathrm{Cu}^{2+}$ centers substituting for Li-ions resulting in a nonzero net ferroelectric polarisation $\mathbf{P} \| a$-axis only for $a b$-planar spin spirals, and a zero net effect both for $a c$ - and $b c$-planar spin spirals. Such a behavior differs sharply from the predictions based on the spin current scenario (see Exp.(11)), but it is strictly confirmed in experiments $[1,2]$ where the ferroelectric polarisation in $\mathrm{LiVCuO}_{4}$ has been observed only along $a$-axis irrespective of the direction of the external magnetic field. Our model predicts the spontaneous electric polarisation $P_{a}$ is proportional to the $m^{2}(T), m(T)$ being the magnetic order parameter, which agrees with the experimental findings [2]. The model reproduces nicely a smooth suppression of the effect with increasing external field $\mathbf{h} \| \mathbf{c}$. Given reasonable estimates for the off-diagonal exchange integrals: $I_{b_{1 g} e_{u}(\sigma)} \approx 0.1 \mathrm{eV}$, and for the $g-u$ energy separation: $\Delta_{u g} \approx 4 \mathrm{eV}$, we arrive at an estimate for the maximal value of the electric polarisation per unit cell occupied by an impurity $P \approx 0.1 e \AA$, or $P \approx 0.5 \mu \mathrm{C} / \mathrm{cm}^{2}$ [26]. 
However, the actual net polarisation is strongly reduced compared with this huge magnitude due to the small impurity concentration $c \ll 1$ and the oscillatory character of $P_{a}(y)$. Anyhow, even for reasonable small substitutions $\mathrm{c}\left(\mathrm{Cu}_{L i}\right) \approx 0.03-0.05[20,25]$ and after averaging over impurity positions the net impurity polarisation in $\mathrm{LiVCuO}_{4}$ can still be comparable with that observed in the gigantic multiferroic $\mathrm{Ni}_{3} \mathrm{~V}_{2} \mathrm{O}_{8}$ [6].

Above we assumed a weak coupling between the $\mathrm{Cu}^{2+}$ substituent and the adjacent spin spirals treating the substituent as a paramagnetic impurity. However, the $\mathrm{Cu}^{2+}$ substituent itself can locally distort the spin spirals. Irrespective of the sign of the exchange parameters the $\mathrm{Cu}^{2+}$ substituent - spin spiral exchange interaction favours locally a ferromagnetic inter-chain coupling with a phase shift $\Delta \alpha=0$, thus leading to a inter-chain coupling competing with that of the ideal chain system. The role played by this effect in magnetoelectric coupling deserves a special investigation. Also the effect of an external magnetic field on the ferroelectricity in spiral magnets seems to be more involved than it appears in our simplified model theory, and needs both in a more detailed and sophisticated description of interacting impurity centers, spirals, and a field induced changes of the spirals themselves. In particular, the pecularities near $8 \mathrm{~T}$ observed in the magnetization measurements $[27,28]$ may evidence a breaking of the weak $\mathrm{Cu}^{2+}$ substituent - spin spiral coupling approximation thus making a breakdown of the ferroelectricity observed near $8 \mathrm{~T}[1,2]$ quite natural. Finally, also the effect of finite temperatures should be considered. As regards the quantitative estimates of the net electric polarisation in $\mathrm{LiVCuO}_{4}$ it is worth noting that, strictly speaking, the parity-breaking exchange results in electric polarisation of both exchange-coupled centers with a trend toward a cancellation, if the inversion center takes place inbetween. Indeed, the $\mathrm{Cu}^{2+}$ substituent will polarise the four adjacent $\mathrm{CuO}_{4}$ centers which net polarisation, however, fails to cancel $P_{a}(y)$ due to a nonequivalence of the exchange-coupled $\mathrm{CuO}_{4}$ centers.

Anyhow, we have shown that in contrast to a spincurrent scenario our simplified microscopic model already catches qualitatively correct the main features of the multiferroicity observed in $\mathrm{LiVCuO}_{4}$, namely the $a$-axis direction of the net electric polarisation for the $a b$-plane spin spiral ordering and its vanishing for the $b c$ - and $a c$-planar spirals. Along with a reasonable estimation of the polarization, the model is believed to explain the subtleties of the field dependence, in particular, the suppression of the polarisation with increasing external magnetic field \| $c$-axis.

In conclusion, we have discussed recent observations of the multiferroic behavior in the $1 \mathrm{D}$ chain cuprate $\mathrm{LiVCuO}_{4}$ with the edge-shared $\mathrm{CuO}_{4}$ plaquettes and incommensurate spin ordering and argued that its peculiarities can be consistently explained if one takes into account the exchange-induced electric polarisation on the $\mathrm{Cu}^{2+}$ centers substituting for $\mathrm{Li}$-ions in $\mathrm{LiVCuO}_{4}$. Thus we deal with an unusual system where in sharp contrast with manganites the multiferroicity happens in two different subsystems, that is magnetic spirals induce electric polarisation at $\mathrm{Cu}^{2+}$ ions substituting for native $\mathrm{Li}^{+}$ ions. These substituent centers are proved to be an effective probe of the spin incommensurability and magnetic field effects. The experimental investigation of possible relations between physical properties and the extent of nonstoichiometry as well as the theoretical consideration of analogous scenarios in other multiferroics would be of considerable scientific and potential practical interest.

Note added. After the submission of the manuscript, we became aware of an independent experimental study of the multiferroicity in $\mathrm{LiVCuO}_{4}$ by Schrettle et al. [29]. In contrast with Yasui et al. [2], the authors have found a weak $c$-axis electric polarization also for a $b c$-plane spin spiral. Generally speaking, this discrepancy does not necessarily contradict the nonstoichiometric nature of the multiferroic behaviour which makes it strongly sample dependent. In the general framework of an impurity model this might point to a manifestation of weak charge transfer effects between the $\mathrm{Cu}^{2+}$ substituent and the spin spirals. Anyhow, further theoretical and experimental studies are needed to uncover all the subtle details of the unusual and challenging multiferroicity in $\mathrm{LiVCuO}_{4}$.

\section{$* * *$}

We thank Yu. Panov, A. Loidl, U. Rößler, K. Dörr, R. Kuzian and H. Rosner for discussions. The DFG and RFBR Grants (Nos. 06-02-17242, 06-03-90893, and 07-0296047) are acknowledged for financial support.

\section{REFERENCES}

[1] Naito Y. et al., J. Phys. Soc. Jpn., 76 (2007) 023708

[2] YASUI Y. et al., arXiv:cond-mat/0711.1204, (2007)

[3] PARK S. et al., Phys. Rev. Lett., 98 (2007) 057601

[4] Katsura H. et al., Phys. Rev. Lett., 95 (2005) 057205

[5] Mostovoy M., Phys. Rev. Lett., 96 (2006) 067601

[6] Lawes G. et al., Phys. Rev. Lett., 95 (2005) 087205

[7] Chapon L.C. et al., Phys. Rev. Lett., 96 (2006) 097601

[8] Betouras Joseph J. et al., Phys. Rev. Lett., 98 (2007) 257602

[9] Sergienko I.E. et al., Phys. Rev. Lett., 97 (2006) 227204

[10] Sergienko I.E. and Dagotto E., Phys. Rev. B, 73 (2006) 094434

[11] Tanabe Y. et al., Phys. Rev. Lett., 15 (1965) 1023

[12] Tanaka S. et al., Phys. Rev. Lett., 97 (2006) 116404

[13] Hu C.D., arXiv:cond-mat/0608470, (2006)

[14] JIA C. et al., arXiv:cond-mat/0701614, (2007)

[15] Nakajima T. et al., J.Phys. Soc. Jap., 76 (2007) 043709

[16] Moskvin A.S. and Drechsler S.-L., arXiv:condmat/0710.0496, (2007)

[17] Moskvin A.S., JETP, 104 (2007) 911

[18] Moskvin A.S., Phys. Rev. B, 75 (2007) 054505

[19] We note that $\mathrm{LiVCuO}_{4}$ is not a vanadate but a lithium vanadyl cuprate. For this reason we prefer the short notation $\mathrm{LiVCuO}_{4}$ instead of the standard one $\mathrm{LiCuVO}_{4}$. 
[20] Prokofiev A.V. et al., Journal of Solid State Chemistry, 177 (2004) 3131

[21] Brown I.D. and D. Altermatt, Acta Crystallogr. Sect. B: Struct. Sc., 41 (1985) 244

[22] Gibson B.J. et al., Physica B, 350 (2004) e253

[23] Enderle M. et al., Europhys. Lett., 70 (2005) 237

[24] Büttgen N. et al., ArXiv:cond-mat/0701083v1, (2007)

[25] Kegler C. et al., Phys. Rev. B, 73 (2006) 104418

[26] A partial cancellation $(\sim 60 \%)$ should be made as actually the $b_{2 g}$ orbital is a superposition of $\mathrm{Cu} 3 d$ and $\mathrm{O} 2 p$ contributions.

[27] Drechsler S.-L. et al., J. of Magnet. \& Magnet. Mat., 316 (2007) 306

[28] Blanks M. et al., J. Phys.-Cond. Mat., 19 (2007 ) 145227

[29] Schrettle F. et al., arXiv:cond-mat/0712.3583v1, (2007) 